\title{
Hypofractionated intensity-modulated radiotherapy in patients with localized prostate cancer: a preliminary study
}

\author{
Hye Jin Kang, MD', Chul-Seung Kay, MD', Seok Hyun Son, MD', \\ Myungsoo Kim, MD', In Young Jo, MD', So Jung Lee, MD', \\ Dong Hwan Lee, MD², Hong Jin Suh, MD², Yong Sun Choi, MD² \\ Departments of ${ }^{1}$ Radiation Oncology and ${ }^{2} U$ rology, Incheon St. Mary's Hospital, \\ The Catholic University of Korea College of Medicine, Incheon, Korea
}

Purpose: The aim of this work was to assess the efficacy and tolerability of hypofractionated intensity-modulated radiotherapy (IMRT) in patients with localized prostate cancer.

Materials and Methods: Thirty-nine patients who received radical hypofractionated IMRT were retrospectively reviewed. Based on a pelvic lymph node involvement risk of 15\% as the cutoff value, we decided whether to deliver treatment prostate and seminal vesicle only radiotherapy (PORT) or whole pelvis radiotherapy (WPRT). Sixteen patients (41\%) received PORT with prostate receiving $45 \mathrm{~Gy}$ in $4.5 \mathrm{~Gy}$ per fraction in 2 weeks and the other 23 patients (59\%) received WPRT with the prostate receiving $72 \mathrm{~Gy}$ in $2.4 \mathrm{~Gy}$ per fraction in 6 weeks. The median equivalent dose in $2 \mathrm{~Gy}$ fractions to the prostate was $79.9 \mathrm{~Gy}$ based on the assumption that the $\alpha / \beta$ ratio is $1.5 \mathrm{~Gy}$.

Results: The median follow-up time was 38 months (range, 4 to 101 months). The 3-year biochemical failure-free survival rate was $88.2 \%$. The 3 -year clinical failure-free and overall survival rates were $94.5 \%$ and $96.3 \%$, respectively. The rates of grade 2 acute genitourinary (GU) and gastrointestinal (GI) toxicities were 20.5\% and 12.8\%, respectively. None of the patients experienced grade $\geq 3$ acute $\mathrm{GU}$ and $\mathrm{GI}$ toxicities. The grade $2-3$ late $\mathrm{GU}$ and $\mathrm{Gl}$ toxicities were found in $8.1 \%$ and $5.4 \%$ of patients, respectively. No fatal late toxicity was observed.

Conclusion: Favorable biochemical control with low rates of toxicity was observed after hypofractionated IMRT, suggesting that our radiotherapy schedule can be an effective treatment option in the treatment of localized prostate cancer.

Keywords: Prostate cancer, Intensity-modulated radiotherapy, Disease-free survival, Toxicity

\section{Introduction}

Early detection of localized prostate cancer has dramatically increased because of the widespread use of screening tests. Prostate cancer becomes the second most common malignancy worldwide in men, accounting for $15 \%$ of the total number of newly diagnosed cancer cases [1]. In Korea, prostate cancer is the fifth most common male malignancy, and approximately 9,000 men are newly diagnosed with prostate cancer every year [2].

Radical radiotherapy is the treatment of choice for localized prostate cancer. In several randomized trials, escalated-dose

Received 8 September 2015, Revised 6 February 2016, Accepted 10 March 2016.

Correspondence: Chul-Seung Kay, MD, Department of Radiation Oncology, Incheon St. Mary's Hospital, The Catholic University of Korea College of Medicine, 56 Dongsu-ro, Bupyeong-gu, Incheon 21431, Korea. Tel: +82-32-280-6075, Fax: +82-32-280-6024, E-mail: k41645@chol.com

(C) This is an Open Access article distributed under the terms of the Creative Commons Attribution Non-Commercial License (http://creativecommons.org/ licenses/by-nc/4.0/) which permits unrestricted non-commercial use, distribution, and reproduction in any medium, provided the original work is properly cited.

www.e-roj.org 
radiotherapy was superior to conventional-dose radiotherapy for biochemical control in prostate cancer patients [3-7]. However, prolongation of the waiting period, increases in the overall treatment time, and high health care costs are inevitable in high-dose delivery by conventional fractionation. These drawbacks can be overcome by hypofractionation that delivers a fractionated dose of $>2 \mathrm{~Gy}$. Also, the value of the $\alpha /$ $\beta$ ratio for prostate cancer was reported to be approximately $1.5 \mathrm{~Gy}$, which is lower than the value of organs at risk (OARs) associated with late toxicity [8-11]. These findings support the therapeutic advantage of hypofractionation.

Intensity-modulated radiotherapy (IMRT) allows for hypofractionation and shortening of the overall treatment time by delivering differential doses to multiple target organs [12]. Moreover, a reduction in normal tissue volume receiving IMRT may deliver high dose radiation to the prostate with no increase in late toxicity [13]. Therefore, IMRT is an effective method for the radical treatment of localized prostate cancer.

In the present study, we retrospectively reviewed the efficacy and tolerability of hypofractionated IMRT in patients with localized prostate cancer.

\section{Materials and Methods}

\section{Study patients}

Between August 2007 and October 2014, a total of 39 patients with prostate cancer were retrospectively included in the study. Inclusion criteria were as follows: 1) histological confirmation of prostate adenocarcinoma; 2) pelvic lymph node (LN) enlargement $<1 \mathrm{~cm}$ on computed tomography (CT) or magnetic resonance imaging; 3) no evidence of distant metastasis; 4) no history of pelvic radiotherapy; 5) no history of prostate surgery other than transurethral resection; 6) no history of radical radiotherapy; 7 ) equivalent dose in $2 \mathrm{~Gy}$ fractions $\left(\mathrm{EQD}_{2 \mathrm{~Gy}}\right)$ to the prostate $\geq 72 \mathrm{~Gy}$ when the $\alpha / \beta$ value was assumed to be $1.5 \mathrm{~Gy}$; and 8) patients with $>3$ months of follow-up.

The patient characteristics are shown in Table 1. The median age of 39 patients was 68 years (range, 49 to 88 years). The distributions of Gleason score, T stage, and prostate specific antigen (PSA) for all patients are listed in Table 1. Patients were divided into the intermediate-risk (46.2\%) and highrisk (53.8\%) groups according to the National Comprehensive Cancer Network (NCCN) prostate cancer risk classification. The median follow-up time was 38 months (range, 4 to 101 months).

\section{Radiotherapy}

For simulation and treatment, patients were placed in the supine position with their hands placed on the anterior chest. A whole-body vacuum cushion and an abdominal compression were used for immobilization. Planning CT scans in 2.5$\mathrm{mm}$ thickness were obtained from the lower abdomen to the pelvis. The patients were requested to keep their rectum empty through daily defecation. For patients with habitual constipation, a light laxative was administered before the start of radiotherapy. The patients were instructed to void their bladder at least 2 hours before the simulation and treatment.

The target volume was delineated on CT images. Clinical target volume 1 (CTV1) encompassed the prostate and the base of the seminal vesicle. CTV2 encompassed CTV1 with isotropic $5-\mathrm{mm}$ margin in all directions and the remaining distal portion of the seminal vesicle. CTV3 encompassed the common iliac, internal/external iliac, presacral, and obturator LN chains only for patients at high risk of pelvic LN involvement. The planning target volume (PTV) was created by adding an isotropic $3-\mathrm{mm}$ margin to CTV. The portion of target overlapping with the rectum was excluded from the target volume. In addition, OARs (rectum, bladder, femoral heads, and intestinal loops)

Table 1. Patient characteristics

\begin{tabular}{lc}
\hline \multicolumn{1}{c}{ Characteristic } & No. of patients (\%) \\
\hline Total no. of patients & 39 \\
Age (yr), median (range) & $68(49-88)$ \\
EQD $_{2 \text { Gy }}$ (Gy), median (range) & $79.9(77.1-92.9)$ \\
Gleason score & \\
$4-6$ & $14(35.9)$ \\
7 & $9(23.1)$ \\
$8-10$ & $16(41.0)$ \\
PSA (ng/mL) & $18(46.2)$ \\
$<10$ & $10(25.6)$ \\
$10-20$ & $11(28.2)$ \\
$>20$ & \\
T stage & $2(5.1)$ \\
1 & $25(64.1)$ \\
2 & $25(64.1)$ \\
3 & $1(2.6)$ \\
4 & \\
Risk group & $21(53.8)$ \\
High & $18(46.2)$ \\
Intermediate & \\
TURP & $2(5.1)$ \\
Yes & $37(94.9)$ \\
No & \\
\hline
\end{tabular}

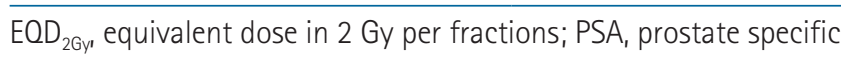
antigen; TURP, transurethral resection of prostate. 
were contoured. The rectum was delineated from the anal verge to the rectosigmoid junction. The intestinal loop encompassed the small intestine and colon from the upper margin of the rectum to $2.5 \mathrm{~cm}$ above PTV.

Based on a pelvic LN involvement risk of $15 \%$ as the cutoff value using the Roach formula, 2 types of hypofractionated treatment schedule were used whether pelvic LN was included in the target or not. The prostate doses of both treatment modalities used in this study were based on the linearquadrantic formula in order to achieve a biologically equivalent dose (BED) of $81 \mathrm{~Gy}$. The BED for $81 \mathrm{~Gy}$ in $1.8 \mathrm{~Gy}$ per fraction was $178.2 \mathrm{~Gy}$ when the $\alpha / \beta$ value was assumed to be $1.5 \mathrm{~Gy}$. In the prostate and seminal vesicle only radiotherapy (PORT) group of 16 patients (41.0\%), the median prescription doses for PTV1 and PTV2 were 45 Gy (range, 45 to 50 Gy) and $40 \mathrm{~Gy}$ (range, 40 to $45 \mathrm{~Gy}$ ) in 10 fractions in 2 weeks, respectively. In the whole pelvis radiotherapy (WPRT) group of 23 patients (59\%), the median prescription doses for PTV1, PTV2, and PTV3 were 72 Gy (range, 67.5 to $79.2 \mathrm{~Gy}$ ), 66 Gy (range, 60 to $72.6 \mathrm{~Gy}$ ), and $54 \mathrm{~Gy}$ (range, 50.4 to $60 \mathrm{~Gy}$ ) in 30 fractions in 6 weeks, respectively. The dose distributions of the 2 treatment modalities are shown in Fig. 1. The median as $E Q D_{2 G y}$ to the prostate was $79.9 \mathrm{~Gy}$ (range, 77.1 to 92.9) when the $\alpha / \beta$ value was assumed to be $1.5 \mathrm{~Gy}$.

The dose for the $95 \%$ of PTV $\left(D_{95 \%}\right)$ was determined as the prescribed dose or higher. The constraints for the OARs were defined as follows. For the PORT group, the rectal constraint was defined as at least $30 \mathrm{~Gy}\left(\mathrm{~V}_{30 \mathrm{~Gy}}\right) \leq 30 \%$ and $40 \mathrm{~Gy}\left(\mathrm{~V}_{40 \mathrm{~Gy}}\right) \leq$ $15 \%$. The bladder constraints were defined as $V_{30 G y}<50 \%$. For the WPRT group, the rectal constraint was defined as $V_{50 G y}<$ $30 \%$ and $V_{70 G y}<15 \%$. The bladder $V_{40 G y}$ would be $\leq 50 \%$. The femoral head were planned to $V_{506 y}<10 \%$.

The treatment planning system TomoTherapy Hi-Art System ver. 4.0.4 (Accuray Inc., Sunnyvale, CA, USA) was used to generate IMRT. Treatment was conducted using the TomoTherapy Hi-Art system. Megavoltage CT was scanned daily for imageguided verification before treatment.

\section{Androgen deprivation therapy}

Approximately 59\% of the patients received androgen deprivation therapy (ADT). ADT consisted of a combination of antiandrogen and luteinizing hormone-releasing hormone agonist. The patients in the intermediate-risk group were treated with short-term ADT for 4-6 months, and those in the high-risk group received long-term ADT for 2-3 years.
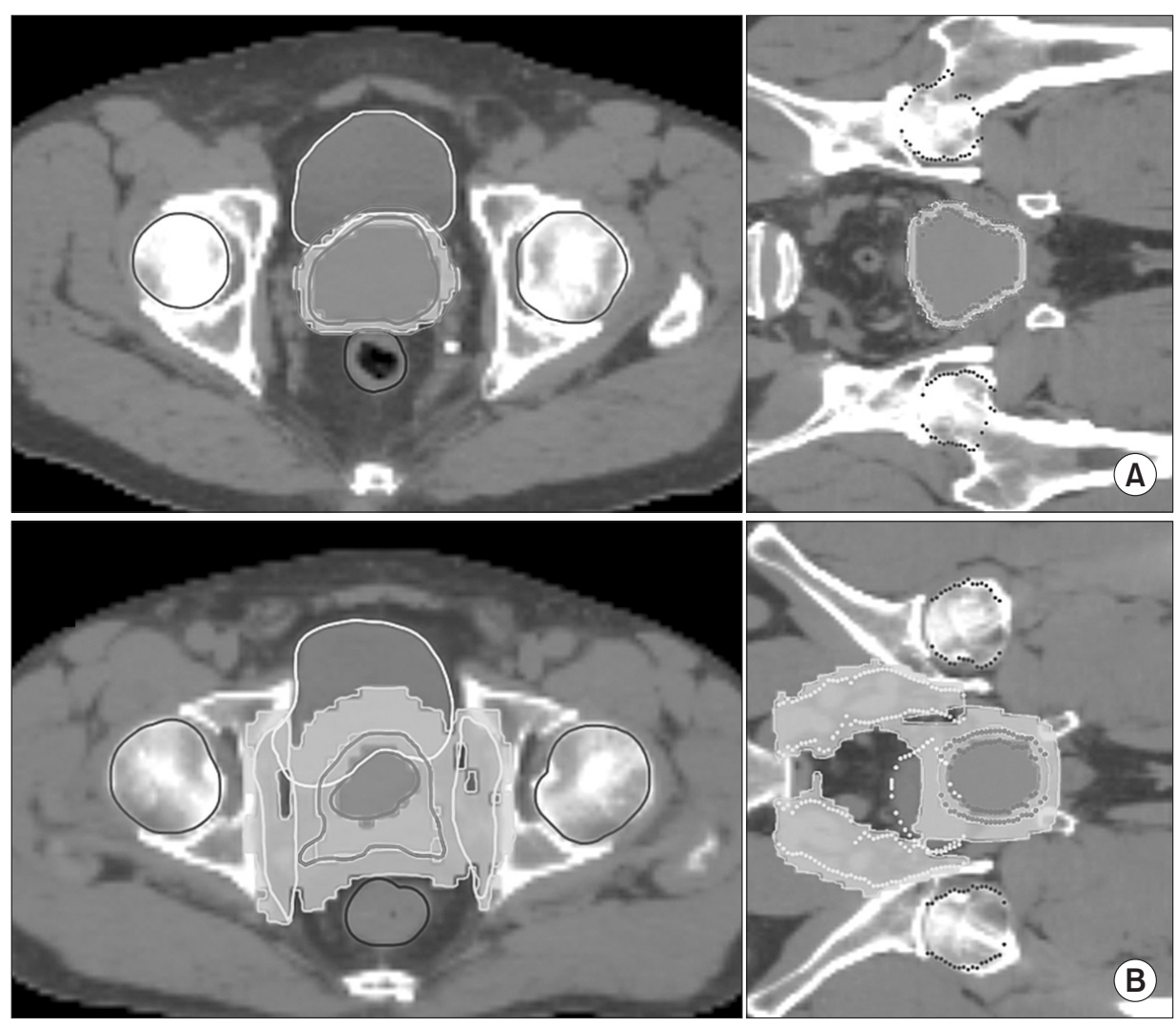

B

http://dx.doi.org/10.3857/roj.2016.34.1.45
Fig. 1. Dose distribution on transverse and coronal slices of (A) PORT and (B) WPRT. Planning was carried out as described in the Materials and Methods section. (A) PORT plan shows planning target volume (PTV) coverage by 45-Gy and 40-Gy isodose lines. (B) WPRT plan shows PTV coverage by 72-Gy, 66-Gy, and 54Gy isodose lines. PORT, prostate and seminal vesicle only radiotherapy; WPRT, whole pelvis radiotherapy. 


\section{Follow-up and endpoints}

Patients were scheduled to be seen weekly during radiotherapy and followed up after treatment at 1 month after the end of treatment, every 3 months for the first 2 years, and every 6 months thereafter. Physical examination and PSA assay were performed at each visit. Abdominal and pelvis CT and bone scan were done in cases of suspected recurrences or metastases.

The primary endpoint was biochemical failure-free survival (BFFS). Biochemical failure was defined as a PSA increase of $\geq 2 \mathrm{ng} / \mathrm{mL}$ from nadir after radiotherapy (also known as the Phoenix definition). The secondary endpoints were clinical failure-free survival (CFFS), overall survival (OS), and toxicity. Clinical failure was defined as both locoregional and distant failures.

Radiotherapy-related toxicity was scored according to the Common Terminology Criteria for Adverse Events (CTCAE) 4.0 criteria. Toxicity was recorded on the basis of severity at the time of follow-up, regardless of the duration of symptoms. The pre-existing symptoms before treatment were excluded to correctly evaluate the toxicity. Acute toxicity was scored weekly during radiotherapy, and 1 and 3 months after completion of the treatment. Late toxicity was recorded at each visit for 6 months after the end of radiotherapy.

\section{Statistical analysis}

All time points were calculated from the date of initial diagnosis. For all endpoints measured, patients were censored at the time of specific events related to prostate cancer. The survival curves of BFFS, CFFS, and OS were calculated by using the KaplanMeier estimates. The hazard ratios of clinical factors affecting

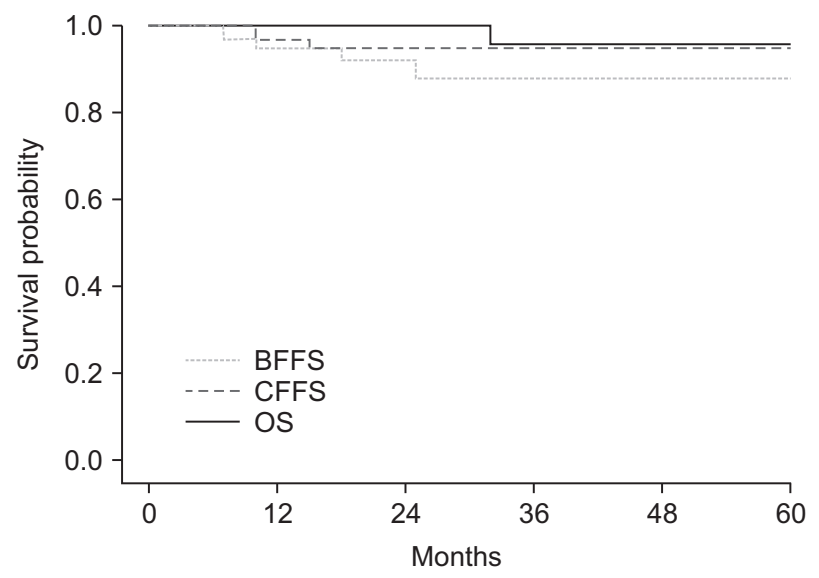

Fig. 2. Kaplan-Meier estimates of disease-free survival. BFFS, biochemical failure-free survival; CFFS, clinical failure-free survival; OS, overall survival. cancer control were analyzed by using the Cox proportional hazards model. All the test results were two-sided. A p-value of less than 0.05 was considered statistically significant. All statistical analyses were done with $R$ (ver. 3.2.1).

\section{Results}

\section{Cancer control}

For all the patients, the 3-year BFFS was $88.2 \%$ (Fig. 2). For patients in the intermediate-risk and high-risk groups, the 3-year BFFS were $87.1 \%$ and $90.5 \%$, respectively. Two patients in each group were confirmed as biochemical failure by regular PSA follow-up and received salvage ADT. The 3-year CFFS was 94.5\% (Fig. 2). Two cases of clinical failures were observed only in the high-risk group, and both of them showed distant bone metastases without locoregional failure. The 3-year OS for all the patients was $96.3 \%$ (Fig. 2). Only one case of death was observed, which was a cancer-specific death. There was no statistically significant prognostic factor affecting BFFS, CFFS, or OS.

\section{Acute toxicity}

The proportion of patients with genitourinary (GU) symptoms before radiotherapy was 53.9\% (21 patients). Most of the patients had grade 1 irritative symptoms, such as frequency and nocturia due to prostate hyperplasia. One patient had grade 2 urinary incontinence due to pre-existing cervical spinal cord injury. The cumulative incidence of grade 2 acute GU toxicity was $20.5 \%$. None of the patients experienced grade $\geq 3$ acute GU toxicity.

The proportion of baseline gastrointestinal (GI) symptoms was 10.3\% (four patients). The rate of developing grade 2 acute $\mathrm{Gl}$ toxicity was $12.8 \%$. None of the patients experienced grade $\geq 3$ acute $\mathrm{Gl}$ toxicity. The distributions of acute $\mathrm{GU}$ and $\mathrm{Gl}$ toxicities according to the treatment field are shown in Table 2.

Table 2. Incidences of toxicity $\geq$ grade 2

\begin{tabular}{ccc}
\hline & PORT & WPRT \\
\hline Acute toxicity & & \\
Genitourinary & $1(2.6)$ & $7(17.9)$ \\
Gastrointestinal & $1(2.6)$ & $4(10.2)$ \\
Late toxicity & & \\
Genitourinary & $0(0)$ & $3(8.1)$ \\
Gastrointestinal & $0(0)$ & $2(5.4)$ \\
\hline
\end{tabular}

Values are presented as number (\%).

PORT, prostate and seminal vesicle only radiotherapy; WPRT, whole pelvis radiotherapy. 


\section{Late toxicity}

During the entire follow-up period, the cumulative incidence of grade $\geq 2$ late GU toxicity was $8.1 \%$ (three patients): grade 2 toxicity was observed in one patient, and grade 3 toxicity in two patients. No grade 4 toxicity was observed. The grade 2 toxicity was observed in only one patient who had persistent dysuria. The two patients with grade 3 toxicity had gross hematuria requiring hospitalization and transfusion, which, resolved after treatment. Therefore, the rate of developing grade $\geq 2$ late GU toxicity was $2.7 \%$ at the last follow-up.

For all the follow-up patients, the cumulative incidence of grade $\geq 2$ late $\mathrm{Gl}$ toxicity was $5.4 \%$ (two patients). The two patients had grade 2 toxicity. No grade 3 or 4 late Gl toxicity was observed. Two late $\mathrm{Gl}$ toxicities were rectal bleeding that required endoscopic intervention. In one patient, the bleeding was stopped immediately after intervention, and in the other patient rectal bleeding continued after intervention for 1 year. No grade $\geq 2$ late Gl toxicity was observed at the last followup. All the grade $\geq 2$ late toxicities were observed in patients who received WPRT. The distributions of late GU and GI toxicities according to the treatment field are shown in Table 2.

\section{Discussion and Conclusion}

Numerous phase III randomized trials on escalated-dose radiotherapy for localized prostate cancer compared to conventional-dose radiotherapy were conducted in the 1990s, and the long-term results have been reported. Escalated-dose radiotherapy has been demonstrated to improve biochemical control in prostate cancer patients [3-7]. The MD Anderson dose-escalation trial increased the prescribed dose from 70 to $78 \mathrm{~Gy}$ in 301 patients [6]. They showed that an absolute benefit of 19\% in the 10-year BFFS rate and a greater benefit of $39 \%$ was seen in patients with initial PSA $>10 \mathrm{ng} / \mathrm{mL}$. Also, the 10-year CFFS rate was significantly increased in the highdose arm compared to the low-dose arm (85\% vs. 93\%, p $=0.0014)$. The advantage of escalated-dose radiotherapy in biochemical control was confirmed in patients with prostate cancer in all the risk groups by The Medical Research Council RT01 trial [3]. NCCN recommends that the dose of 75.6-79.2 Gy for the low-risk group and the dose of up to 81 Gy for the intermediate-risk or high-risk group in conventional fractions should be used to improve biochemical control [14].

However, high-dose delivery of up to 75.6-81 Gy by conventional fractionation of 1.8-2 Gy per fraction increases the overall treatment time to $8-9$ weeks, waiting periods, and health care costs. These drawbacks can be overcome by hypofractionation that delivers a fraction size greater than $2 \mathrm{~Gy}$ with a lower overall dose, which shortens the treatment time to achieve equivalent efficacy to conventional fractionation [8]. Also, more important advantage of hypofractionation is therapeutic benefit. Prostate cancer has a slow growth fraction and a low estimated $\alpha / \beta$ ratio of approximately 1.5 Gy; however, for normal tissues adjacent to the prostate, such as the bladder and rectum the $\alpha / \beta$ value was assumed to be 3-5 Gy. The tissues with a low $\alpha / \beta$ ratio are more sensitive to fraction size, suggesting the therapeutic advantage of hypofractionation [8-11]. The randomized trial with the aim of exploring the efficacy of hypofractionation was conducted by Arcangeli et al. $[15,16]$. They treated high-risk prostate cancer patients and compared conventional fractionation (80 Gy/40 fractions) to hypofractionation (62 Gy/20 fractions). The 3-year BFFS rates were $79 \%$ in the control group and $87 \%$ in the hypofractionation group $(p=0.035)$. At a median follow-up of 70 months, the absolute reduction in biochemical failure by hypofractionation was $10.3 \%(p=0.011)$. In our study, 39 patients with intermediate-risk or high-risk prostate cancer received high-dose radiation at $\mathrm{EQD}_{2 \mathrm{~Gy}}$ of 79.9 Gy using hypofractionated IMRT. In contrast to most randomized studies on dose escalation and hypofractionation, we treated the pelvic LN in 59\% of patients at high-risk of pelvic LN involvement. The effect of elective pelvic LN chain irradiation is not clear because pelvic LN chain coverage without substantial toxicity is insufficient in the conventional technique $[17,18]$. However, the prescribed dose to the pelvic LN chain in our study was escalated to $54 \mathrm{~Gy}$ that led to good regional control. This is a possible explanation of good biochemical control of highrisk patients in our study. The 3-year BFFS rate of all patients was reported to be $88.2 \%$. For intermediate-risk and high-risk patients, the 3-year BFFS were $87.1 \%$ and $90.5 \%$, respectively. Guckenberger et al. [19] treated 150 prostate cancer patients with hypofractionated IMRT at EQD 2 Gy of 79.9-82.4 Gy $(\alpha)$ $\beta=1.5 \mathrm{~Gy}$ ), of whom 41 simultaneously received pelvic $L N$ irradiation. With a median follow-up of 50 months, the 3 - and 5 -year BFFS rates were 95\% and $82 \%$, respectively, and the 3 -year BFFS rates were $95 \%$ of intermediate-risk patients and $89 \%$ of high-risk patients, which are similar to our results.

High-dose radiotherapy improves biochemical control, but it increases risk of treatment-related normal tissue damage $[3,5,6,20]$. The Memorial Sloan-Kettering Cancer Center evaluated the difference in toxicity rate between prescribed doses of 64.8-81 Gy in 743 patients with localized prostate cancer [20]. Significantly increased late toxicity rates were identified in patients treated with higher radiation doses. The 
incidences of grade $\geq 2$ late $\mathrm{GU}$ and $\mathrm{GI}$ toxicities were $8 \%$ and $7 \%$, respectively, in the lower-dose group (64.8-70.2 Gy) compared to $15 \%$ and $16 \%$, respectively, in the higher-dose group (75.6-81 Gy) ( $p=0.004)$.

Normal tissue complication, especially GI toxicity, can be reduced by the use of IMRT that helps save OARs from highdose exposure. Zelefsky et al. [21] reported the incidence of late toxicity in 741 patients receiving IMRT compared to 830 patients receiving three-dimensional conformal radiation therapy (3D-CRT). The incidence of GI and GU toxicities was increased in patients receiving high radiation doses of 66-81 $\mathrm{Gy}$, whereas that of $\mathrm{Gl}$ toxicity was significantly reduced in patients receiving IMRT. With a median follow-up of 10 years, the incidence of grade $\geq 2$ late GI toxicity was significantly reduced in patients receiving IMRT compared to those receiving 3D-CRT (5\% vs. 13\%, $p<0.001$ ). The incidence of late GU toxicity in all the patients was 15\%. The risk of late GU toxicity was related to the prescribed dose regardless of receiving 3D-CRT or IMRT ( $<81$ Gy, 12\% vs. 81 Gy, 20\%; $p=$ 0.01). Similar results were reported by Jani et al. [13]. Rectum and bladder volumes was significantly lower in patients receiving mid to high dose $\left(\mathrm{V}_{40-70 \mathrm{G}}\right)$ IMRT than in those receiving 3D-CRT. IMRT substantially reduces the incidence of grade 1 late $\mathrm{Gl}$ toxicity $(p<0.001)$, and the incidence of grade $\geq 2$ late GI toxicity was $6 \%$ in the IMRT group. However, there was no significant difference in the incidence of GU toxicity ( $p=0.166$ ) between the study groups. A possible explanation of this result may be that urinary symptoms originate from the urethra which frequently overlaps with the GTV $[22,23]$. Both IMRT and 3D-CRT are not specifically constrained by the urethra. Even though, the incidence of late GU toxicity showed acceptable considering irradiation dose and most of the symptoms were transient.

In our study, the incidences of grade $\geq 2$ late GU and $\mathrm{Gl}$ toxicities in all the patients were $8.1 \%$ and $5.4 \%$, respectively. Although we employed high-dose radiotherapy in patients with prostate cancer was similar to low-dose radiotherapy. Moreover, 16 patients who received PORT had no grade $\geq 2$ late toxicity. The late toxicity of hypofractionated IMRT was reported by Guckenberger et al. [19] who treated 150 prostate cancer patients including 41 patients receiving pelvic LN irradiation. The total dose of prostate was $\mathrm{EQD}_{2 \mathrm{~Gy}}$ of 79.9-82.4 Gy $(\alpha / \beta=1.5$ Gy). With a median follow-up of 50 months, the incidence of grade $\geq 2$ late GU toxicity was $22.8 \%$ and not significantly different between PORT and WPRT; the incidence of grade $\geq 2$ late $\mathrm{Gl}$ toxicity was below $5 \%$.

In this study, we found that during a median follow-up of
38 months and a maximum of 101 months, hypofractionated IMRT delivered high dose radiation to the prostate and pelvic LN chain and resulted in good biochemical cancer control with acceptable toxicity. The results of this study suggest that hypofractionated IMRT can be an effective method for the treatment of localized prostate cancer and that our radiotherapy schedule may be effective and safe to perform in patients planned for PORT of small treatment fields because it shortens the overall treatment time to 2 weeks. Further studies with a longer follow-up period are required to confirm our results.

\section{Conflict of Interest}

No potential conflict of interest relevant to this article was reported.

\section{References}

1. World Cancer Research Fund International. Worldwide data: world cancer statistics for the most common cancers [Internet]. London: World Cancer Research Fund International; c2015 [cited 2015 Aug 12]. Available from: http://www.wcrf.org/int/ cancer-facts-figures/worldwide-data.

2. Jung KW, Won YJ, Kong HJ, et al. Cancer statistics in Korea: incidence, mortality, survival, and prevalence in 2012. Cancer Res Treat 2015;47:127-41.

3. Zietman AL, DeSilvio ML, Slater JD, et al. Comparison of conventional-dose vs high-dose conformal radiation therapy in clinically localized adenocarcinoma of the prostate: a randomized controlled trial. JAMA 2005;294:1233-9.

4. Peeters ST, Heemsbergen WD, Koper PC, et al. Dose-response in radiotherapy for localized prostate cancer: results of the Dutch multicenter randomized phase III trial comparing $68 \mathrm{~Gy}$ of radiotherapy with 78 Gy. J Clin Oncol 2006;24:1990-6.

5. Dearnaley DP, Jovic G, Syndikus I, et al. Escalated-dose versus control-dose conformal radiotherapy for prostate cancer: long-term results from the MRC RT01 randomised controlled trial. Lancet Oncol 2014;15:464-73.

6. Kuban DA, Tucker SL, Dong L, et al. Long-term results of the M. D. Anderson randomized dose-escalation trial for prostate cancer. Int J Radiat Oncol Biol Phys 2008;70:67-74.

7. Eade TN, Hanlon AL, Horwitz EM, Buyyounouski MK, Hanks GE, Pollack A. What dose of external-beam radiation is high enough for prostate cancer? Int J Radiat Oncol Biol Phys 2007; 68:682-9.

8. Ray KJ, Sibson NR, Kiltie AE. Treatment of breast and prostate cancer by hypofractionated radiotherapy: potential risks and benefits. Clin Oncol (R Coll Radiol) 2015;27:420-6. 
9. Tucker SL, Thames HD, Michalski JM, et al. Estimation of alpha/ beta for late rectal toxicity based on RTOG 94-06. Int J Radiat Oncol Biol Phys 2011;81:600-5.

10. Miralbell R, Roberts SA, Zubizarreta E, Hendry JH. Dosefractionation sensitivity of prostate cancer deduced from radiotherapy outcomes of 5,969 patients in seven international institutional datasets: $\alpha / \beta=1.4(0.9-2.2)$ Gy. Int J Radiat Oncol Biol Phys 2012;82:e17-24.

11. Proust-Lima C, Taylor JM, Secher $S$, et al. Confirmation of a low $\alpha / \beta$ ratio for prostate cancer treated by external beam radiation therapy alone using a post-treatment repeatedmeasures model for PSA dynamics. Int J Radiat Oncol Biol Phys 2011;79:195-201.

12. Dolezel $M$, Odrazka $K$, Zouhar $M$, et al. Comparing morbidity and cancer control after 3D-conformal (70/74 Gy) and intensity modulated radiotherapy (78/82 Gy) for prostate cancer. Strahlenther Onkol 2015;191:338-46.

13. Jani $A B$, Su A, Correa D, Gratzle J. Comparison of late gastrointestinal and genitourinary toxicity of prostate cancer patients undergoing intensity-modulated versus conventional radiotherapy using localized fields. Prostate Cancer Prostatic Dis 2007;10:82-6.

14. National Comprehensive Cancer Network. NCCN guidelines for patients: prostate cancer [Internet]. Fort Washington, PA: National Comprehensive Cancer Network; c2016 [cited 2016 Mar 17]. Available from: http://www.nccn.org/patients/ guidelines/prostate/files/assets/common/downloads/files/ prostate.pdf.

15. Arcangeli S, Strigari L, Gomellini S, et al. Updated results and patterns of failure in a randomized hypofractionation trial for high-risk prostate cancer. Int J Radiat Oncol Biol Phys 2012; 84:1172-8.
16. Arcangeli G, Saracino B, Gomellini S, et al. A prospective phase III randomized trial of hypofractionation versus conventional fractionation in patients with high-risk prostate cancer. Int J Radiat Oncol Biol Phys 2010;78:11-8.

17. Huang $E H$, Pollack $A$, Levy $L$, et al. Late rectal toxicity: dosevolume effects of conformal radiotherapy for prostate cancer. Int J Radiat Oncol Biol Phys 2002;54:1314-21.

18. Yeoh $E_{1}$ Horowitz $M$, Russo $A$, et al. Effect of pelvic irradiation on gastrointestinal function: a prospective longitudinal study. Am J Med 1993;95:397-406.

19. Guckenberger M, Lawrenz I, Flentje M. Moderately hypofractionated radiotherapy for localized prostate cancer: long-term outcome using IMRT and volumetric IGRT. Strahlenther Onkol 2014;190:48-53.

20. Zelefsky MJ, Cowen D, Fuks Z, et al. Long term tolerance of high dose three-dimensional conformal radiotherapy in patients with localized prostate carcinoma. Cancer 1999;85:2460-8.

21. Zelefsky MJ, Levin EJ, Hunt M, et al. Incidence of late rectal and urinary toxicities after three-dimensional conformal radiotherapy and intensity-modulated radiotherapy for localized prostate cancer. Int J Radiat Oncol Biol Phys 2008;70: 1124-9.

22. Teh BS, Mai WY, Augspurger ME, et al. Intensity modulated radiation therapy (IMRT) following prostatectomy: more favorable acute genitourinary toxicity profile compared to primary IMRT for prostate cancer. Int J Radiat Oncol Biol Phys 2001;49:465-72.

23. Zelefsky MJ, Chan H, Hunt M, Yamada Y, Shippy AM, Amols $H$. Long-term outcome of high dose intensity modulated radiation therapy for patients with clinically localized prostate cancer. J Urol 2006;176(4 Pt 1):1415-9. 